\title{
La mercantilización del saber científico
}

\author{
Germán Anzola Montero ${ }^{1}$ \\ ${ }^{1}$ Rector. Universidad de Ciencias Aplicadas y Ambientales U.D.C.A, (D) https://orcid.org/0000-0001-6075-2595
}

\begin{abstract}
Como citar: Anzola Montero, G. 2021. La mercantilización del saber científico. Rev. U.D.C.A Act. \& Div. Cient. 24(1):e1911. http://doi. org/10.31910/rudca.v24.n1.2021.1911

Artículo de acceso abierto publicado por Revista U.D.C.A Actualidad \& Divulgación Científica, bajo una licencia Creative Commons CC BY-NC 4.0

Publicación oficial de la Universidad de Ciencias Aplicadas y Ambientales U.D.C.A, Institución de Educación Superior Acreditada de Alta Calidad por el Ministerio de Educación Nacional.
\end{abstract}

¿Pagar por publicar en una revista? O ¿cobrar por descargar artículos científicos? Son interrogantes que han surgido entre las comunidades académicas y científicas del mundo, en especial, para los países en vía de desarrollo, una situación que no debería, ni siquiera, tener consideración alguna.

En el círculo científico y, en especial, en el de las Universidades, que son las que más generan productos científicos y en los centros de investigación, se ha analizado el tema, llegando a la conclusión que los artículos científicos se han convertido en artilugios para hacer dinero.

Un profesor universitario, que cumple funciones de investigación, es pretender publicar un artículo con los resultados científicos obtenidos y es aquí, donde estas revistas científicas, tienen como objetivo brindar un medio para evaluar, publicar y divulgar estudios, investigaciones y otras tipologías científicas, para que su trabajo sea reconocido por otros pares y por la comunidad científica y consolidar el avance de la ciencia; sin embargo, en este proceso es cuando surgen los inconvenientes, porque se alimenta la avaricia y se saca provecho, no beneficio, de la necesidad de publicar.

Hoy por hoy, se habla, por una parte, de los indicadores para medir la calidad de la producción científica, como el factor de impacto (frecuencia con la que una revista ha sido citada en un año concreto), el índice de inmediatez (rapidez con la que se citan artículos de una revista científica y permite identificar publicaciones récord en investigaciones de amplia influencia), el cuartil (indicador de posición de una revista con relación a todas las de su área), el Índice H (indicador que evalúa la producción científica de un investigador y relaciona el número de publicaciones con las citas que suscitan), el Índice G (indicador que también cuantifica la productividad bibliométrica, basada en el historial de publicaciones de los autores), el SCImago Journal Rank, SJR (indicador que analiza el área de investigación, la calidad y la reputación de una revista científica y su impacto directo sobre el valor de la cita) y el Journal Metrics (proporciona los datos del CiteScore y del percentil donde se encuentran posicionadas las revistas, de acuerdo con la información extraída de la base de datos Scopus) (ULPGC, s/f).

Por otra parte, también se deben tener en cuenta, para publicar, los índices de valoración de las publicaciones, que se dividen en: a) indices de calidad relativa de las revistas que, entre otros, son: Journal Citation Reports, JCR, que suministra datos sobre revistas científicas en el campo de las ciencias aplicadas y sociales y forma parte de la Web of Science, pero será el Institute of Scientifc Information, ISI, de Thompson Scientifics, el responsable de analizar las revistas, de elaborar el factor de impacto y difundirlo; Scimago Journal Rank (SJR), un portal que analiza, de las revistas incluidas en Scopus, los índices bibliométricos; Scientific Electronic Library Online, Scielo, que es una biblioteca científica de revistas de América Latina y el Caribe, que incluye datos de editoriales, el factor de impacto, la vida media, las citas recibidas y citas concedidas y otros más; b) criterios de calidad editorial de una revista, como DIALNET (identificar revistas de mayor influencia y confirmar el prestigio de las publicaciones); Sistema Regional de 
Información en línea para revistas científicas de América Latina, el Caribe, España y Portugal, LATINDEX (cuenta con criterios de calidad para las revistas impresas y para revistas electrónicas); Publindex (evaluación y clasificación de revistas científicas nacionales, creado por Colciencias, hoy MinCiencias) y otras de carácter europeo, como TRID, DICE, MIAR, REHS, FECYT, ERIH, CARHUS PLUS y c) criterios de calidad de las editoriales: Book Citation Index (de la base de datos WOS, para saber si un libro está o no indizado y también el listado de editores), Publish or Perish (un programa que busca y analiza citas académicas utilizando Google Scholar) y Agencia Internacional de ISBN, que ofrece enlaces a las Agencias Nacionales de ISBN (Universo escrito, 2019).

Todos estos indicadores y criterios, se han convertido en la cara de la moneda de la ciencia.

El investigador, entonces, cuando tiene su manuscrito científico, lo que se pregunta es dónde puede publicar, puesto que su trayectoria investigativa y el reconocimiento de los resultados obtenidos, hoy por hoy, depende, en gran medida, del número de artículos científicos publicados, las revistas en donde se encuentran y el número de citas recibidas; de esta manera, se crea cierta presión, pues se requiere tener un ranking, tanto de la revista como del artículo y todos estos indicadores y criterios expuestos, le permitirán decidir en dónde publicar.

Primero, tiene que ser una revista especializada, en el área de conocimiento de la investigación, con un factor de impacto alto, en cuartil 1 o 2 de Scopus u otra base de datos reconocida mundialmente y que el tiempo de publicación no sea muy extenso, porque, de lo contrario, los resultados podrían perder vigencia o generar desactualización. A esto, se le suma averiguar la conveniencia de someter el artículo a una determinada revista, dependiendo de la modalidad de financiación adoptada.

Justamente, en esta etapa, es lo que se discute y se analiza la mercantilización del saber científico. Muchas revistas científicas no cobran al autor por publicar; pero si al lector y hasta el mismo autor cuando quieren descargar el artículo u obtener un ejemplar del número. Es lo que se denomina suscripciones a las revistas y es una figura que utilizan las universidades, los centros de investigación e investigadores, para tener acceso a la información científica. El valor de suscripción a las revistas científicas colombianas o latinoamericanas es muy bajo, en comparación con el cobro que tienen, por ejemplo, las revistas europeas o americanas.

En el caso de las revistas que operan con el sistema Open Access (que emerge de la crisis de las publicaciones impresas, por sus altos costos en producción y acceso a Internet), abiertas, disponibles en línea, sin barreras legales y que se puede descargar gratuitamente el artículo en versión PDF, el problema se le traduce al autor, dado que debe pagar altas sumas de dinero para ver publicado su artículo científico (muchas Universidades apoyan económicamente a sus profesores-investigadores para esta gestión). Algunas tarifas, se pueden consultar, sin problema, en Science Advances o PlosONE. Si se mira objetivamente este sistema abierto, sin duda favorecerá exclusivamente a los investigadores, quienes pertenecen a grupos con alta financiación y robustos en producción científica, pues obtendrán grandes ganancias, por la cantidad de artículos publicados (Miró et al. 2013).

Al final de cuentas, para cualquiera de los dos casos, el investigador no gana dinero por la publicación de sus artículos.

Centrémonos en el acceso abierto, un fenómeno que, en la última década, viene creciendo y que se ha convertido en el boom de las publicaciones científicas. Según la Declaración Budapest Open Access Initiative (BOAI, 2002), la que definió, por primera vez, el concepto de acceso abierto y estableció unas condiciones para lograr este sistema. Por una parte, refirió la Via Dorada, que corresponde a la publicación de la revista de acceso abierto y la Via Verde, para el autoarchivo de los artículos en repositorios institucionales, después de la publicación en revistas. Sin duda, este sistema permitió que muchos artículos estuvieran disponibles en acceso abierto, en poco tiempo.

Bajo la Vía Dorada, muchas revistas cobran a los autores una alta tasa por publicar, modelo adoptado por una gran mayoría de editoriales comerciales y algunas sin ánimo de lucro; también, existen revistas que subvencionan la totalidad de la publicación, como las Universidades, como el caso de la U.D.C.A y algunos centros de investigación. Otras revistas, que en el medio las llaman híbridas, que se financian por medio de las suscripciones y ofrecen la open choice, donde los investigadores-autores pueden liberar sus artículos, pero pagando una cuantiosa suma.

Las grandes cinco editoriales científicas que adoptan la Vía Dorada son Elsevier, Springer, Wiley, Tayloy \& Francis y Sage que, además, concentran más del 50\% de los nuevos artículos que se publican, debido a que sus revistas serían las de mayor impacto en la comunidad científica. Esto las ubica como empresas altamente rentables (Luchilo, 2019), ya que cobran por los diferentes servicios que ofrecen, como la prestación del software para la administración de la revista, la diagramación de los artículos en los diferentes formatos, las estadísticas, las asesorías, entre otros; Cabe resaltar que, dentro de estas editoriales mencionadas, se pueden encontrar revistas con todas las modalidades de financiación.

Por su parte, la venta de suscripciones, actual modelo de negocios de otras grandes editoriales, consiste en ofrecer a las instituciones académicas este servicio, para que sus investigadores y estudiantes puedan acceder a las revistas de estas empresas.

El Open Access, por lo tanto, es un tema de interés, que genera desacuerdos entre la comunidad académica y científica, en especial, en lo referente a los aspectos económicos, dado que requieren de fuentes de financiación y que, por obvias razones, la fuente primaria son los autores, ya que que están obligados a publicar los resultados de la investigación y, hasta que no se publique, no existe.

Este esquema, de pagar por publicar, presenta ventajas, traducidas en acceso inmediato a la información científica por cualquier usuario (¿un bien común?), avance de la ciencia a favor de la 
sociedad, rendir cuentas a la sociedad sobre los recursos invertidos en investigación (lo que justifica el quehacer investigativo en las Universidades), artículos más leídos, más compartidos y más citados y rapidez en el proceso editorial; pero también está el contexto negativo: los elevados costos de las publicaciones, que puede generar desigualdades en la oportunidad de publicar; discriminar o relegar a investigadores, en especial, aquellos que pertenecen a los países de América Latina y el Caribe o países en desarrollo; no brindar oportunidad a aquellas investigaciones en áreas de conocimiento que no captan o atraen apoyo financiero; jóvenes investigadores que no tienen cómo costear su publicación.
Cabe mencionar, que muchos investigadores, cuyo idioma nativo no es el inglés, también deben financiar la traducción "oficial" de sus publicaciones, debido a que se considera que la ciencia se escribe en inglés, razón, por la cual, varias revistas científicas solo aceptan que los manuscritos estén en este idioma.

En la actualidad, no se ha podido definir quién debería cubrir estos costos de publicación de un artículo (Tabla 1); algunos asumen, que debería ser el autor; otros, la institución a la que pertenecen y otros, la entidad que financia la investigación. Lo que sí se sabe es que estos precios oscilan entre 300 y 3.000 dólares, por artículo

Tabla 1. Modelos económicos de las primeras cinco revistas del mundo, de Latinoamérica y de Colombia, los costos asumidos por el lector o por el autor y el cuartil en el que se encuentra la revista en Scopus.

\begin{tabular}{|c|c|c|c|c|}
\hline Revistas & Tipo de acceso* & $\begin{array}{c}\text { Costo autor, institución } \\
\text { o fundación que } \\
\text { respalde }\end{array}$ & $\begin{array}{l}\text { Costo lector } \\
\text { por artículo }\end{array}$ & $\begin{array}{l}\text { Cuartil } \\
\text { Scopus }\end{array}$ \\
\hline \multicolumn{5}{|c|}{5 mejores revistas del mundo de acuerdo con Scopus } \\
\hline CA - A Cancer Journal for Clinicians & Acceso abierto dorado & US\$3000 & US\$0 & Q1 \\
\hline $\begin{array}{l}\text { Morbidity and Mortality Weekly Report } \\
\text { (MMWR) }\end{array}$ & Acceso abierto platino & US\$0 & $\mathrm{US} \$ 0$ & Q1 \\
\hline Nature review materials & Acceso por suscripción & US\$0 & US $\$ 8.99$ & Q1 \\
\hline Quarterly Journal of Economics & Acceso por suscripción & US $\$ 0$ & US\$86 & Q1 \\
\hline Nature reviews molecular cell biology & Acceso por suscripción & US\$0 & US\$8.99 & Q1 \\
\hline \multicolumn{5}{|c|}{5 mejores revistas de la región de acuerdo con Scopus** } \\
\hline Perspectives in Ecology and Conservation & Acceso abierto platino & US\$0 & US\$0 & Q1 \\
\hline $\begin{array}{l}\text { Revista Mexicana de Astronomía y } \\
\text { Astrofísica }\end{array}$ & Acceso abierto platino & US $\$ 0$ & US $\$ 0$ & Q1 \\
\hline $\begin{array}{l}\text { International Journal of Educational } \\
\text { Technology in Higher Education }\end{array}$ & Acceso abierto platino & US\$0 & US $\$ 0$ & Q1 \\
\hline Memorias do Instituto Oswaldo Cruz & Acceso abierto platino & US\$0 & US\$0 & Q1 \\
\hline Alea & Acceso abierto platino & US\$0 & US $\$ 0$ & Q1 \\
\hline \multicolumn{5}{|c|}{5 mejores revistas de Colombia de acuerdo con Scopus*** } \\
\hline $\begin{array}{l}\text { International Journal of Educational } \\
\text { Technology in Higher Education }\end{array}$ & Acceso abierto platino & US $\$ 0$ & US $\$ 0$ & Q1 \\
\hline Revista Latinoamericana de Psicología & Acceso abierto platino & US\$0 & US $\$ 0$ & Q2 \\
\hline Ensayos Sobre Política Económica & Acceso abierto platino & US $\$ 0$ & US\$0 & Q1 \\
\hline Kepes & Acceso abierto platino & US\$0 & US $\$ 0$ & Q1 \\
\hline Revista Colombiana de Estadística & Acceso abierto platino & US $\$ 0$ & US\$0 & Q3 \\
\hline
\end{tabular}

Tipo de acceso abierto dorado*: Las revistas no cobran a los lectores por el acceso a los artículos y pueden cobrar o no a los autores, institución o fundación que los respalda, por los cargos de procesamiento de los artículos. Acceso platino: Las revistas no cobran a los lectores por el acceso a los artículos, ni a los autores, institución o fundación que los respalde, por la tasa de procesamiento de los artículos; estas revistas son financiadas por instituciones, fundaciones o sociedades académicas. Acceso abierto híbrido: El acceso a los artículos es por suscripción; sin embargo, los autores pueden pagar para que los lectores tengan acceso abierto a los artículos. Acceso por suscripción: Los lectores pagan por el acceso a los artículos o pueden acceder a los artículos por medio de una institución que paga por el acceso. Cargos de procesamiento de los artículos*: Los CPA, se cobran después de la aceptación del artículo y antes de la publicación y cubren: trabajo editorial, infraestructura técnica e innovación, producción de artículos, comercialización de la revista y del contenido, atención al cliente. De las 50 primeras revistas de la región latinoamericana solo la revista Ameghiniana cobra a los autores, institución o fundación que los respalda por los cargos de procesamiento de los artículos, que en este caso es de US\$300**. De las 50 primeras revistas de Colombia ninguna cobra a los lectores por el acceso a los artículos, ni a los autores por los cargos de procesamiento de los artículos***. 
(según PlosONE), que corresponde a las revistas que tienen altos factores de impacto y son las que buscan los investigadores. El anhelo y la expectativa de la comunidad científica, definitivamente, es que los resultados de la investigación científica sean consultados por cualquier persona, descargados por el interesado y publicados sin tener costo.

En síntesis, buena parte de las investigaciones científicas son subvencionadas, ya sea por las Universidades (en el caso de la U.D.C.A, el 90\% son financiadas por la Institución), por los Centros de Investigación o por el Estado (cuando se accede a las convocatorias), pero lo lamentable del asunto, es que los resultados de estas investigaciones quedan en manos de editoriales comerciales que se lucran de la divulgación del conocimiento científico, al que, solamente, se puede acceder con pago previo de sus publicaciones; sin embargo, la mayoría de los investigadores no son partidarios de esta modalidad y reconocen que la falta de financiación a la investigación es la razón principal para no poder hacer frente a los pagos exigidos por las editoriales comerciales.

La pregunta obligada es ¿cuánto están dispuestos los autores a pagar por la publicación de sus artículos y todo el servicio que reciben, si se tiene en cuenta que, en la actualidad, la mayoría de las revistas son de acceso abierto y operan bajo este modelo y que, además, otros gastos, como impresión y distribución ya no cuentan y la mayoría de pares o evaluadores externos ya no perciben remuneración? Posiblemente, el Open Access sea un buen modelo de negocio, claro, para las editoriales comerciales, dejando dudas en los beneficios para los autores.

Lo importante es saber qué hacer con ese dinero que se recibe que, a bien, debería ser reinversión en investigación, en ofrecer becas de investigación, en movilidad científica y apoyo a jóvenes investigadores.

Habrá un momento, si así continúa el sistema de publicación científica, que entre editoriales comerciales y Universidades y Centros de investigación negocien un precio justo por publicar, acorde con el producto recibido.
Este es un buen momento, para que desde el Ministerio de Ciencia Tecnología e Innovación se abra el debate y considerar este tema, para lograr un acceso abierto, igual de universal, pero con acceso para todos.

\section{REFERENCIAS}

1. BUDAPEST OPEN ACCESS INITIATIVE, BOAI. 2002. Disponible desde Internet en: https://www.budapestopenaccessinitiative.org/read (con acceso el 08/03/2021).

2. LUCHILO, L.J. 2019. Revistas científicas: oligopolio y acceso abierto. Revista Iberoamericana de Ciencia, Tecnología y Sociedad, CTS. 14(40):41-79.

3. MIRÓ, Ò.; BURBANO, P. 2013. El factor de impacto, el índice h y otros indicadores bibliométricos. Anales del Sistema Sanitario de Navarra. 36(3):371-377. https://dx.doi.org/10.4321/S1137-66272013000300001

4. SCIMAGO. s/f. SJR - SCImago Journal \& Country Rank. Disponible desde Internet en: http://www.scimagojr.com (con acceso el 08/03/2021).

5. UNIVERSIDAD DE LAS PALMAS DE GRAN CANARIA, ULPGC. s/f. Indicadores e índices de la producción científica. Disponible desde Internet en: https://biblioteca.ulpgc.es/factor_impacto (con acceso el 18/03/2021).

6. UNIVERSO ESCRITO. 2019. Métricas para la evaluación de revistas científicas. Disponible desde Internet en: https://universoescrito.com/metricas-para-la-evaluacionde-revistas-cientificas/ (con acceso el 18/03/2021). 